\title{
COMPARISON OF FLOW PATTERNS IN THE RADIOCEPHALIC ARTERIOVENOUS FISTULA THROUG IN VITRO AND IN SILICO STUDY
}

\author{
F. C. M. Oliveira ${ }^{\mathrm{a}}$, \\ K. L. Bessa ${ }^{a}$, \\ and R. W. C. Moreira ${ }^{b}$ \\ ${ }^{\text {a }}$ Universidade Federal do Rio Grande do Norte \\ Departamento de Engenharia Mecânica \\ Centro de Tecnologia \\ Avenida Senador Salgado Filho, 300 \\ Lagoa Nova \\ Natal, Rio Grande do Norte, Brasil \\ CEP 59078-970 \\ fah_hc@hotmail.com \\ ${ }^{\text {b }}$ Universidade Federal do Rio Grande do Norte \\ Departamento de Medicina Integrada \\ Centro de Ciências da Saúde \\ Avenida Nilo Peçanha, s/n, Petrópolis \\ Natal, Rio Grande do Norte, Brasil \\ CEP 590712-300 \\ ricardowahnermoreira@gmail.com \\ Received: September 08, 2015 \\ Revised: October 08, 2015 \\ Accepted: November 09, 2015
}

\section{ABSTRACT}

The vascular accesses to hemodialysis patients usually are through arteriovenous fistula (AVF) or synthetic grafts. The analyses of recirculation zones, stagnation points, secondary flow and low and high shear stress are very important points to study for understand the hemodynamic local in arteriovenous fistula. In this work, it was analyzed through computational fluid dynamics the streamlines in AVF and it was compared with the literature results. The flow patterns were visualized under steady and unsteady condition with Reynolds number ranging from 100 up 600. The results obtained from CFD were very similar with the literature results. The flow becomes more chaotic when the Reynolds number increases. Vortex can be seen in anastomosis region, occupying all transversal section of the artery. Thus, the results obtained using CFD look like the results obtained experimentally.

Keywords: CFD, shear stress, anastomoses

\section{NOMENCLATURE}

da artery diameter, $\mathrm{mm}$

danas anastomosis diameter, mm

$\mathrm{d}_{\mathrm{v}} \quad$ vein diameter, $\mathrm{mm}$

$\mathrm{p}$ pressure, $\mathrm{Pa}$

Re Reynolds number a

$\mathrm{t}$ time, $\mathrm{s}$

$\mathrm{V}$ velocity, $\mathrm{m} / \mathrm{s}$

\section{Greek symbols}

\footnotetext{
$\nabla$ gradient operator a

$\mu$ dynamics viscosity, Pa.s

$v \quad$ kinematic viscosity, $\mathrm{m}^{2} / \mathrm{s}$

$\rho$ density, $\mathrm{kg} / \mathrm{m}^{3}$
}

\section{Superscripts}

AVF arteriovenous fistula

\section{INTRODUCTION}

Vascular access should satisfy three criteria: first, it should be suitable for repetitive circulatory access; second, it should allow for a blood flow suitable to conducting high efficiency dialysis, and third, it should minimize treatment complications (Schwab et al., 2001). The vascular access for hemodialysis patients usually is through native arteriovenous fistula (AVF) or synthetic grafts to allow adequate blood flow during the dialysis session (Yerdel et al., 1997). The AVF is created by vascular surgeon when occurs fail in the kidney. After creating the AVF, blood flow rate slowly progresses and reaches an average of $1200 \mathrm{ml}$ min-1 after maturation (Wedgwood et al., 1984). Due this alteration in blood flow under the artery, it needs to respond the new hemodynamic environment creating a normal adaptation or pathological disease. Common complications are formation of stenosis, thrombosis and aneurysms (Van et al., 2005). These complications show tendency to develop at specific sites, thus suggests that the geometry of the fistula 
and the resulting local hemodynamic conditions have a role to play in the aetiology of these disease (Sivanesan et al., 1999).

According to exposed above, it was used the computational fluid dynamic (CFD) to analyses the flow patterns in AVF. To analyze the flow patterns in AVF, the data were obtained from Sivanesan et al. (1999) entitled: Flow patterns in the radiocephalic arteriovenous fistula: an in vitro study. The comparison of the flow patterns were performed between the planar illuminations of micro-particles suspended in the fluid obtained experimentally (Sivanesa et al., 1999) and the streamlines obtained in sílico. Steady and pulsatile flow studies were performed over a range of flow conditions corresponding to those recorded in patients. Thus, the aim of this paper is understand a little bit the flow patterns in the radiocephalic arteriovenous fistula and their possible contribution to the development of stenosis using CFD.

\section{METHODS}

As mentioned above, the data used for the simulation were obtained from Sivanesan et al. (1999). The choice for AVF is due to their longer operating, the low rate of complications and easy to care for these complications (Bhandari et al., 1995; Santiago-Delphin et al., 1987; Slater and rafter, 1987).

\section{Model Geometry}

The model was based on the geometrical data obtained from bi-planar fistulograms (Sivanesan et al., 1999). The diameter of the vein and artery segments was 7.0 and $4.5 \mathrm{~mm}$, respectively (diameter ratio of 1.6) and the anastomosis angle was $30^{\circ}$ (Figure 1a). In this work and as a first approach, all vessel walls were assumed to be rigid and the blood flow was considered Newtonian, incompressible and homogenous. Tridimensional mesh was constructed in AVF. Tetrahedral mesh was developed in the artery and in the vein with a refining in the anastomosis region, coming down to 390.012 volumes (Figure 1b). The mesh was refined until the flow parameters did not change any further, or rather the results presented in this investigation are meshing independent.

\section{Numerical Model and Flow Conditions}

Mathematic governing equations for incompressible flow and according to the literature (Fox et al., 2006), are presented below:

Mass conservation equation:

$$
\nabla \cdot \vec{V}=0
$$

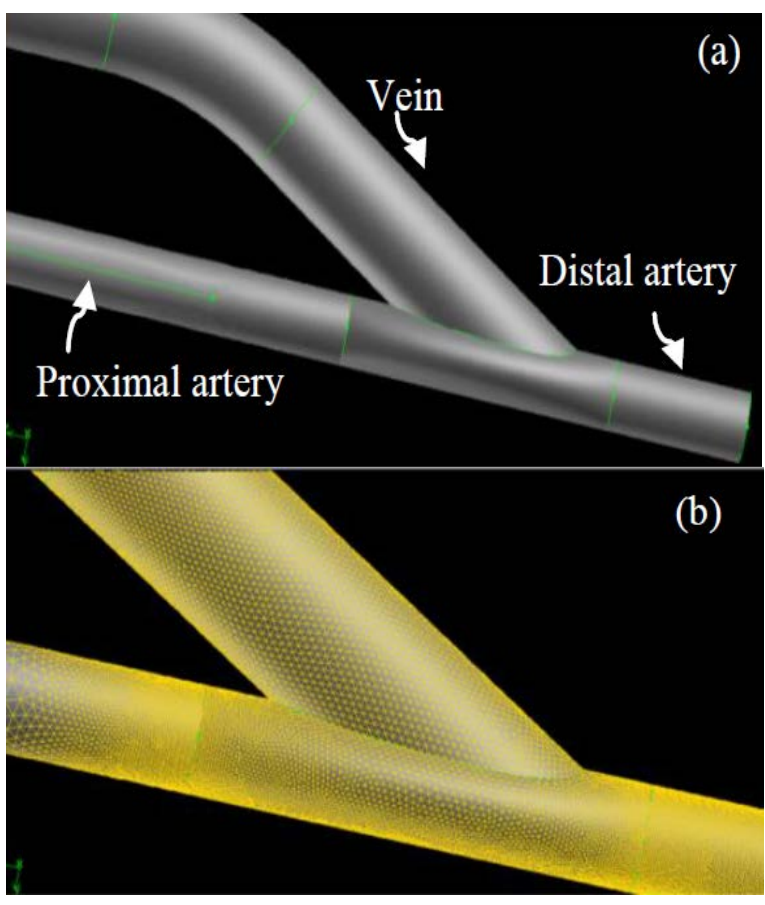

Figure 1. Schematic representation of an AVF: (a) end-to-side model; (b) tetrahedral mesh representation.

Momentum conservation equation:

$$
\rho\left(\frac{D \vec{V}}{D t}\right)=-\nabla p+\mu \nabla^{2} \vec{V}
$$

The CFD code based on the finite volume method (Fluent 6.2.16 Academic License) was used in this study. Discretization of the governing equations at each control volume involved a first order upwind differencing scheme. The resulting system of algebraic equation was solved iteratively using a procedure based on the semi-implicit SIMPLE algorithm. In the AVF model, the inlet velocity profiles were maintained uniform across the inlet cross section. The outlet condition requires that velocity gradients in flow direction be zero. This condition is acceptable due to the outlet boundary having been located far downstream from the junction. The no slip condition was applied to all the walls. Fluid properties were assumed according to the literature (Sivanesan et al., 1999): $\rho=1190 \mathrm{~kg} / \mathrm{m}^{3}$ and $\mu=6.783 \mathrm{mPa}$.s. These values were altered due the refractive index of tube in experimental model (Sivanesan et al., 1999). The flow patterns were visualized under steady and unsteady flow condition with Reynolds number ranging from 100 to 600. For unsteady flow condition the used velocity waveform is showed in the Figure 2. This figure is typical of those recorded in actual AVF. The specific points I, II and III showed in the cycle (Figure 2) are those used to plot the results. The proximal to distal artery flow ratio of 3:1. 


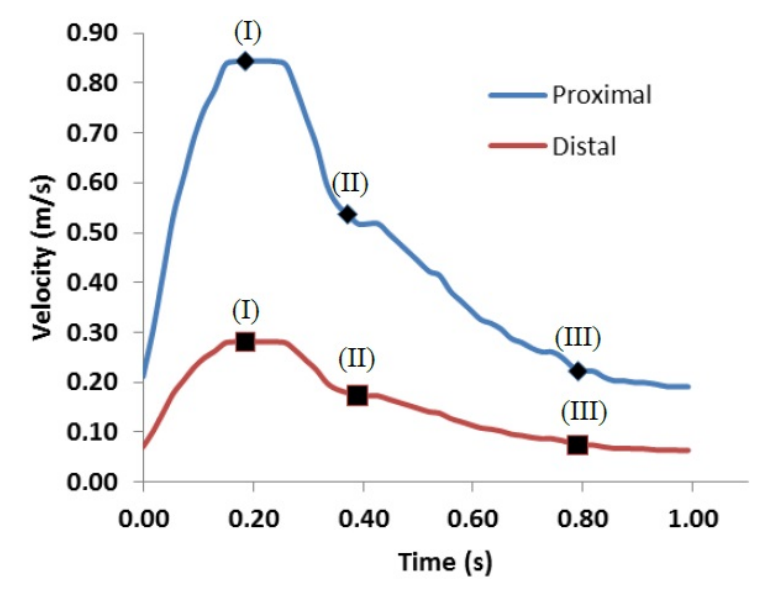

Figure 2. Velocity waveform obtained from intraoperative recording patients.

The wall shear stresses were plotted on the floor of the artery between the following values: -10 to 10 $\mathrm{mm}$ relative to the center of the anastomosis according to Figure 3.

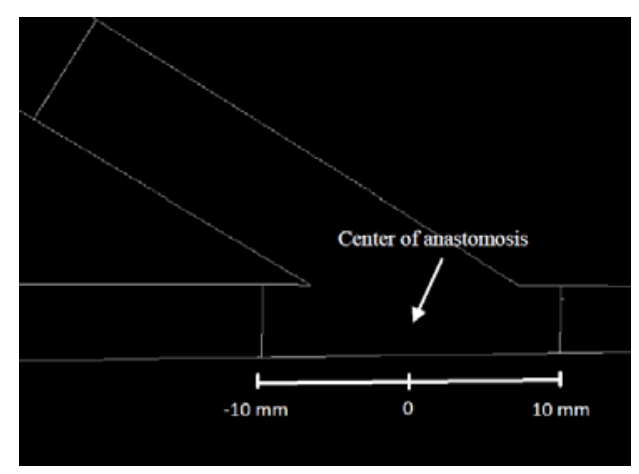

Figure 3. Velocity waveform obtained from intraoperative recording patients.

\section{RESULTS}

The Figures 4 and 5 show the steady flow patterns in the $y-z$ and $x-z$ at Reynolds number of 100,300 and 600 in the vein, with the distal artery occluded and PI of 0.44 . At the artery inlet as determined by mass balance calculations. These figures show a comparison of the flow visualization presented by Sivanesan et al. (1999) and those obtained in this work by computational fluid dynamics.

\section{Steady Flow - Streamlines}

The Figures 4a, 4c and 5a show visualization flow obtained from Sivanesan et al. (1999). The Figures $4 \mathrm{~b}, 4 \mathrm{~d}$ and $5 \mathrm{~b}$ show visualization flow obtained through CFD used in this work. There is a similarity in the Figures $4 \mathrm{a}$ and $4 \mathrm{~b}$. In this figure, the Reynolds number is 100 and the artery velocity inlet is $0.197 \mathrm{~m} / \mathrm{s}$. The flow patterns are highly stable.
When the flow enters in the anastomosis, it separates at the heel where a vortex with a anticlockwise rotation can be seen. When viewed in the $\mathrm{x}-\mathrm{z}$ plane, the vortices can be seen to be symmetrical about the mid plane of the artery. The Figures $4 c$ and $4 d$ for Reynolds number 300 and the velocity inlet 0.590 $\mathrm{m} / \mathrm{s}$, the flow patterns are very similar. Besides that, the fluid motion becomes increasingly chaotic. The Figures $5 \mathrm{a}$ and $5 \mathrm{~b}$ show a disorganized structure in the artery and in the vein, in this case, the artery velocity inlet is $1.182 \mathrm{~m} / \mathrm{s}$.

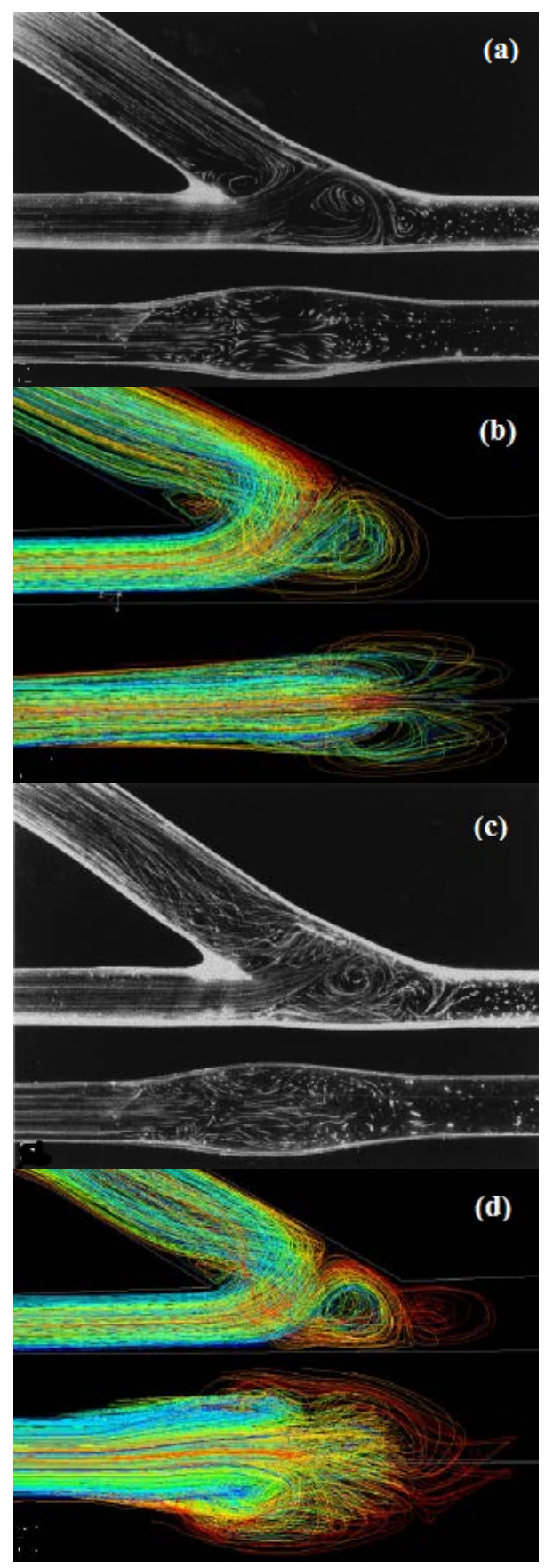

Figure 4. Flow patterns in the planes $y-z$ (top) and $x-z$ (bottom) under steady flow conditions with the distal artery occluded: (a) and (b) $\mathrm{Re}=100$ and (c) and (d) $\operatorname{Re}=300$. 

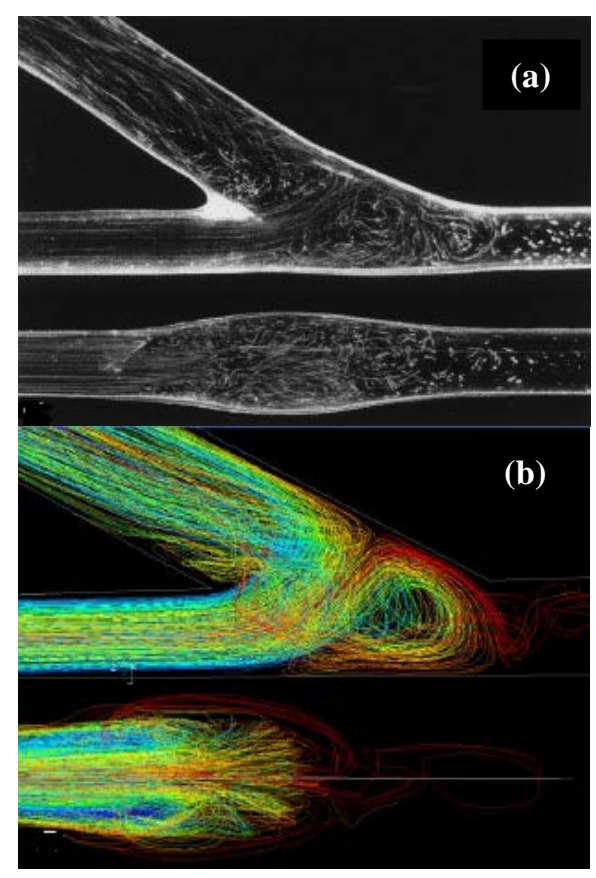

Figure 5. Flow patterns in the planes $\mathrm{y}-\mathrm{z}$ (top) and $\mathrm{x}-\mathrm{z}$ (bottom) under steady flow conditions with the distal artery occluded: (a) and (b) $\mathrm{Re}=600$.

\section{Unsteady flow - streamlines}

The Figures 6 and 7 shows the flow patterns at three specific points in the cycle (proximal to distal artery flow ration $3: 1$, period of the cycle of 1 s). It can be seen that in the specific point I there is not vortices in anastomosis region. However, in the specific points II and III, the vortices appear and the helical movement in the vein appears in these times. The main difference appears to occur in the vortex of the anastomosis region, it fluctuate with the flow.

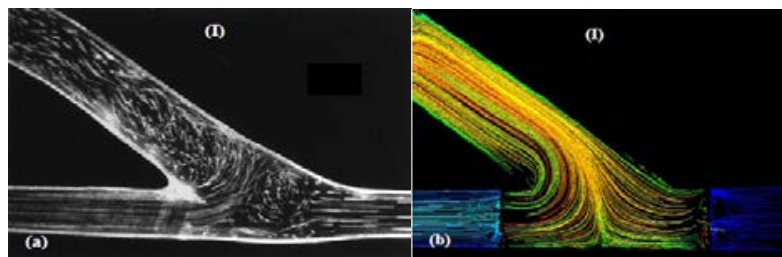

Figure 6. Pulsatile flow patterns at one specific point in the cyclo (ratio of proximal-to-distal artery flow was 3:1): a was withdrawn from Sivanesan et al.,

(1999) and b is result obtained in this paper.

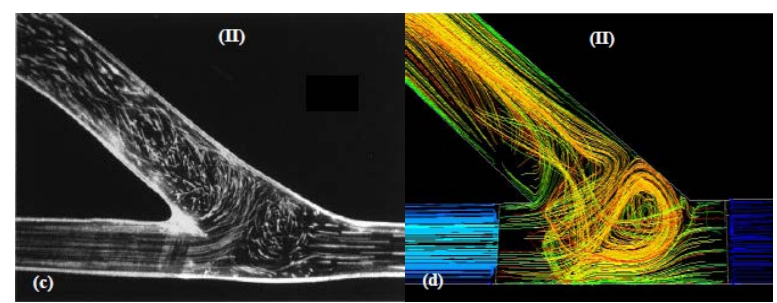

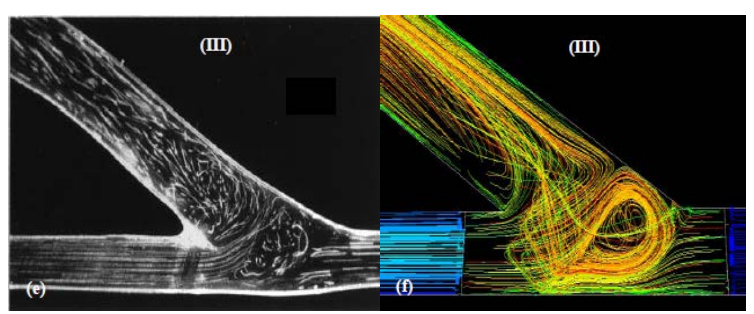

Figure 7. Pulsatile flow patterns at two specific points in the cycle (ratio of proximal-to-distal artery

flow was 3:1): c and e were withdrawn from

Sivanesan et al., (1999) and d and f are result obtained in this paper.

\section{Wall Shear Stress on the Artery Floor}

The Figure 8 shows the values of wall shear stresses along the artery floor in the range from -10 to $10 \mathrm{~mm}$ relative of center of anastomosis. It can be seen the mean and oscillatory values of wall shear stresses during the cycle. The oscillatory values of wall shear stress are those maximum and minimum values occurred for all cycle. The vertical bars denote the maximum and minimum values of wall shear stress. The magnitudes of the mean and oscillatory wall shear stress are highest within the anastomosis, reaching peak values of $14 \mathrm{~Pa}$.

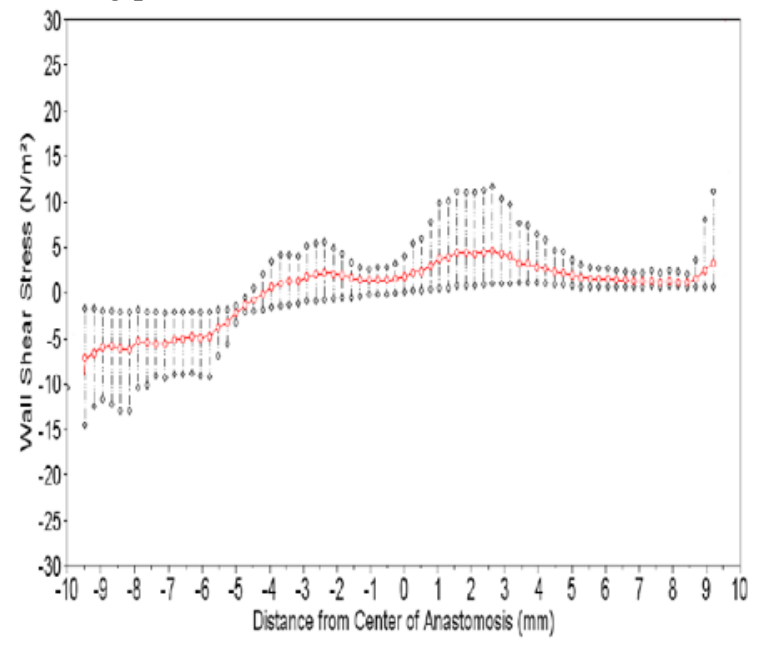

Figure 8. Wall shear stress along of artery floor.

\section{DISCUTION}

In this work, it was analyzed several situations of the flow during the cycle obtained from intraoperative patients. The steady and unsteady flows were analyzed through the flow patterns and the distribution of wall shear stress on the artery floor.

The hemodynamic condition is strongly indicated as a major factor for vascular remodeling and neointima formation (Salam et al., 1996). Flow disturbances and turbulence have been indicated to influence intimal-medial thickening (Fillinger et al., 1989) and endothelial cell turnover (Davies et al., 
1986). Local blood flow conditions seem to be important also in atherosclerosis plaque formation (Nerem, 1991). Studies show that when wall shear stress is low and oscillating, and there are zones of shear stress spatial gradients, vessel walls are more prone to vascular damage. The creation of AVF leads to a complex hemodynamic conditions. The shunt created in AVF alters the blood movement and increase blood flow rate. The blood flow change rapidly of the direction and as result can cause changes in the vascular wall responsible for vascular remodeling, narrowing or dilation of the venous side (Bogdan et al., 2001).

The Figures 4, 5, 6 and 7 show the flow patterns in AVF under steady and unsteady conditions. The flow pattern is very similar between them, but it can be seen that the vortices fluctuate during the cycle. The appearance and disappearance of the vortex leads to regions of recirculation and stagnation points in the anastomotic region. The shear stress presents an oscillatory behavior on the artery floor. Possibly, this oscillatory behavior may disrupt endothelial cells, making this region more susceptible to the formation of atherosclerotic plaques. It is necessary to improve the boundary conditions used in this article so that we can better discuss the influence of hemodynamic forces on the vessel wall.

\section{REFERENCES}

Bhandari, S., Wilkinson, A., and Sellars, L., 1995, Saphenous Vein Forearm Grafts and Gortex thigh Grafts as Alternative forms of Vascular Access, Clinical Nephrology, Vol. 44, No. 5, pp. 325-328.

Ene-Lordache, B., Lidia, M., Giuseppe, R., and Andrea, R., 2001, Computational Fluid Dynamics of a Vascular Access case for Hemodialysis, Journal of Biomechanical Engineering, Vol. 123, No. 3, pp. 284-292.

Davies, P. F., Remuzzi, A., Gordon, E. J., Dewey, C. F., Jr., and Gimbrone, M. A., Jr., 1986, Turbulent Fluid Shear Tress Induces Vascular Endothelial Cell Turnover in Vitro, Proceedings of the National Academy of Sciences of the United States of America, Vol. 83, No. 7, pp. 2114-2117.

Fillinger, M. F., Reinitz, E. R., Schwartz, R. A., Resetarits, D. E., Paskanik, A. M., and Bredenberg, C. E., 1989, Beneficial Effects of Banding on Venous Intimal-Medial Hyperplasia in Arteriovenous Loop Grafts, American Journal of Surgery, Vol. 158, No. 2, pp. 87-94.

Fox, R. W., Pritchard, P. J., and McDonald, A. T., 2006, Introdução à Mecânica dos Fluidos, $7^{\text {a }}$ ed., LTC. (in Portuguese)

Nerem, R. M., 1991, Vascular Fluid Mechanics, the Arterial Wall, and Atherosclerosis, Journal of Biomechanical Engineering, Vol. 114, No. 3, pp. 274-282.

Salam, T. A., Lumsden, A. B., Suggs, W. D., and Ku, D. N., 1996, Low Shear Stress Promotes Intimal Hyperplasia Thickening, Journal Vascular
Investigation, Vol. 2, No. 1, pp. 12-22.

Santiago-Delpin, E. A., Buselmeier, T. J., Simmons, R. L., Najarian, J. S., Kjellstrand, C. M., 1987, A Modified Saphenous Vein Loop-Fistula in the thigh as Vascular Access for Hemodialysis in Patients with Access Problems, Annals of The Royal College of Surgeons of England, Vol. 70, No. 2, pp. 244-245.

Schwab, S. J., Oliver, M. J., Suhocki, P., and McCann, R., 2001, Hemodialysis Arteriovenous Access: Detection of Stenosis and Response to Treatment by Vascular Access Blood Flow, Kidney International, Vol. 59, No. 1, pp. 358-362.

Sivanesan, S., How, T. V., Black, R. A. and Bakran, A., 1999, Flow Patterns in the Radiocephalic Arteriovenous Fistula: an in Vitro Study, Journal of Biomechanics, Vol. 32, No.9, pp. 915-925.

Slater, N. D., and Raftery, A. T., 1987, An Evaluation of Expanded Polytetrafluorethylene (PTFE) Loop Grafts in the thigh as Vascular Access for Hemodialysis in Patients with Access Problems, Annals of The Royal College of Surgeons of England, Vol. 70, No. 3, pp. 244-245.

Van, T. I., De, W. D., Tordoir, J., Verdonck, P., 2005, Hemodynamics and Complications Encountered with Arteriovenous Fistulas and Grafts as Vascular Access for Hemodialysis: a Review, Annals of Biomedical Engineering, Vol. 33, No. 9, pp. 1142-1157.

Wedgwood, K. R., Wiggins, P. A., Guillou, P. J., 1984, A Prospective Study of End-to-Side vs. Side-to-Side Arteriovenous Fistulas for Haemodialysis, British Journal Surgery, Vol. 71, No. 8, pp. 640-642.

Yerdel, M. A., Kesenci, M., Yazicioglu, K. M., Do“seyen, Z., Tu“rkcapar, A. G., and Anadol, E., 1997, Effect of Haemodynamic Variables on Surgically Created Arteriovenous Fistula Flow, Nephrology Dialysis Transplantation, Vol. 12, No. 8, pp. 1684-1688. 